\title{
TFT-LCD Mura Defects Using Independent Component Analysis*
}

\author{
Shang Liang CHEN** and Chi Chin YANG ** \\ ${ }^{* *}$ Institute of Manufacturing Engineering, National Cheng Kung University \\ No. 1, University Road, Tainan City, 701, Taiwan, ROC \\ E-mail:slchen@mail.ncku.edu.tw
}

\begin{abstract}
Independent component analysis (ICA) is used to detect the mura regions with varying sizes and brightness levels before thresholding, then individually analyzed the mura regions in order to avoid unnecessary background effect. Defects detection is performed by partitioning test image into overlapping sub-windows and Classifying each sub-window as normal or mura region by comparing the difference of spatial distance between ICs of defective and non-defective. During the experimental process, a median filter and a high-pass filter are also respectively used to filter out the noise and enhance mura gray intensity. In this research, we developed ICA to achieve off-line learning and on-line detection.
\end{abstract}

Key words: TFT-LCD, Independent Component Analysis, Mura

\section{Introduction}

Mura means the regions or stripes caused by non-uniform brightness and color on a TFT-LCD display panel. They are usually appeared as low contrast, tiny color differences, and non-uniform brightness regions, typically larger than several pixels. Mura defects are hard to be detected by conventional vision inspection. The display quality of TFT-LCD is a major lesson in the final inspection because of various types of defects as line defects, dot defects or mura defects on the LCD panel. These jobs are usually performed by human vision inspection, which also have drawbacks including the tiredness of human vision, inconsistent detection and high cost. Machine vision can improve many of these drawbacks. Hence, independent component analysis (ICA) is used to detect mura defects on the LCD panel in this research. According to definition of SEMI (2002) [1], mura is a non-uniform brightness region which differs form the background and can be identified by human eyes. Fig. 1 shows mura defects. Mura inspection system combines machine vision with technologies such as image processing and pattern recognition.
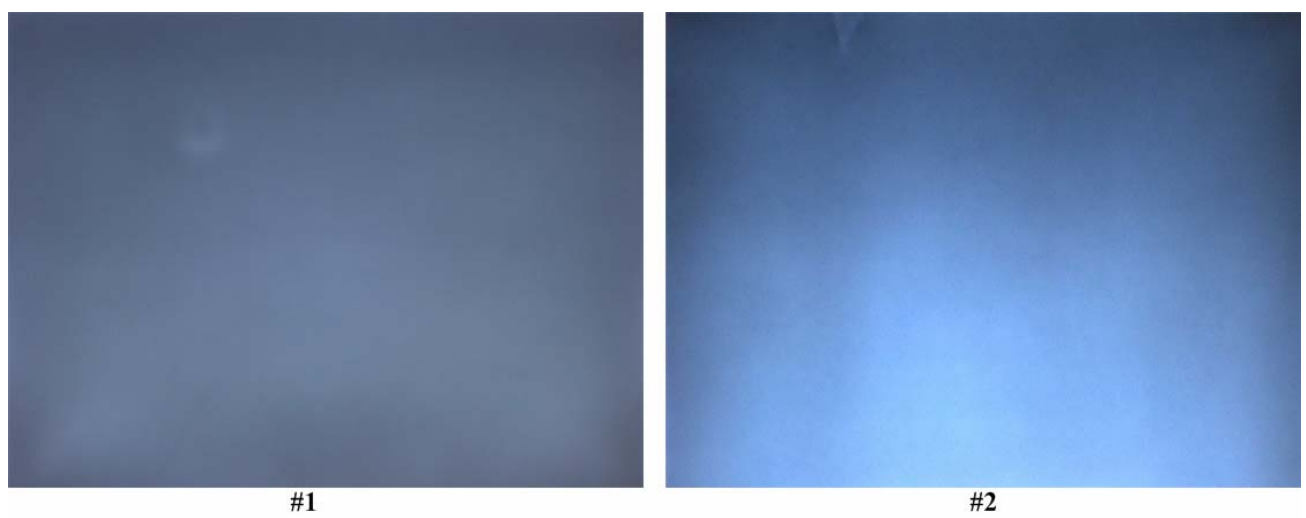

Fig. 1. Mura images (image size: $1200 \times 1600$ ) 
In the research of mura inspection, Lee et al. (2004) [2] proposed Polynomial Background Surface Estimation for inspection on mura defects. The candidate region-muras are segmented from TFT-LCD panel images using the modified regression diagnostics and Niblack's thresholding. The next part is based on the human eye's sensitivity to add mura evaluation for segmenting real muras from candidate muras. Nakano et al. (2005)[3] presented multi-wavelet analysis for detection and quantitative measurement of low-contrast mura.

In this paper, we used ICA to segment mura regions from LCD panel image first, then individually to analyze mura regions in order to avoid unnecessary background effect. In these years, ICA has been developed in image processing including face recognition, texture segmentation and defects inspection. Lu (2004)[4] applied ICA in a non-defective training image to determine the de-mixing matrix and its corresponding independent components (ICs). After identifying a set of proper ICs that represent the global structure of the LCD panel, the corresponding rows of the de-mixing matrix for the identified ICs are replaced with de-mixing rows associated with the least global background information to reform the de-mixing matrix. The reformed de-mixing matrix only containing uniform information of the panel image is used to reconstruct the TFT-LCD image under inspection. Jenssen et al. (2003)[5] presented as a computational technique for creating a new data dependent filter bank for using in texture segmentation. The ICA filters are able to capture the inherent properties of texture images. In this paper, we classify each region as normal or defective by comparing the difference of spatial distances between IC of non-defective and defective. As Amet et al. (1998)[6] applied wavelet transformation to texture defect inspection. Detection of defects within the inspected texture is performed by partitioning the texture image into non-overlapping sub-windows and classifying each sub-window as defective or non-defective with a Mahalanobis distance classifier being trained on defect free samples a priori. Sezer et al. (2004)[7] applied ICA to fabric texture defects detection and classified each sub-window as normal or defective with a Euclidean distance classifier.

\section{Independent Component Analysis Overview}

Assume $x$ is an observed n-dimensional random variables, which are modeled as linear combination of n-dimensional variables $s$, as shown in Equation (1). $s$ (often described as independent components, ICs) is latent variables that can not be directly observed. In ICA model, we usually assume $s$ is statistically independent and the mixing matrix $A$ is unknown, and we through a de-mixing matrix $W$ to transform $x$ to yield independent components, as shown in Equation (2).

$$
x=A s=\sum_{i=1}^{n} a_{i} s_{i}
$$

Where $a_{i}$ is the column vector of mixing matrix $A$ and $s_{i}$ is the row vector of $S$.

$$
y=W x
$$

First we must through two pre-processing steps to remove correlations of data before estimating the de-mixing matrix $W$. The first step is to center $x$ by subtracting its mean vector $E[x]$ so as to make $x$ zero-mean variables. The next step is through principle component analysis to reduce the dimension of the data as we do the whitening using Equation (4).

$$
\begin{gathered}
x=x-E[x] \\
z=V_{P C A} x, V_{P C A}=D^{-1 / 2} E^{T}
\end{gathered}
$$


Where $D$ is the diagonal matrix of eigenvalues of the covariance matrix $E\left[x x^{T}\right]$ and $E$ is the orthogonal matrix of eigenvectors corresponding to its eigenvalues. This means that we have zero-mean, unit variance and uncorrelated variables after this two pre-processing steps, then the ICA problem is to find a de-mixing matrix $W$ to separate ICs such that statistical dependencies between estimated ICs are minimized. Now Equation (2) becomes Equation (5) because of the pre-processing step.

$$
y=W z=W D^{-1 / 2} E^{T} x
$$

In this research, we used FastICA algorithm proposed by Hyvarinen (1999)[8] according to the approximation negentropy. The FastICA algorithm is based fixed-point iteration to find the maximum of $w^{T} z$ such that $y$ of negentropy is maximized, where $w^{T}$ is the row vector of $W$.

\section{Inspection method}

Mura defect image which its background is formed with brightness information is taken as experiment image shown in Fig. 1. The sub-images partitioned from non-defective image are used as the training samples in ICA training algorithm and reduced the dimensionality of the data by using PCA pre-processing step. The independent components and de-mixing matrix can be obtained after the iteration of FastICA algorithm. Similarity, the de-mixing matrix will be used to extract ICs of the incoming test sub-images using Equation (5). Classify each test sub-images as defective or non-defective with spatial distance between ICs of non-defective and defective (Amet et al. (1998)[6] \& Sezer et al. (2004)[7]). The research can be divided into two parts as offline learning (ICA training) and online inspection (ICA detection) described as below:

\subsection{ICA training}

step 1. First, the column vectors of $x$ are formed with $k$ non-overlapping sub-images ( size $n \times n$ ) selected from non-defective image described as below:

$$
x=\left[\begin{array}{cccc}
x_{11} & x_{12} & \cdots & x_{1 k} \\
x_{21} & x_{22} & \cdots & x_{2 k} \\
\vdots & \vdots & \ddots & \vdots \\
x_{n^{2} 1} & x_{n^{2} 2} & \cdots & x_{n^{2} k}
\end{array}\right]=\left[\begin{array}{llll}
x_{1} & x_{2} & \ldots & x_{k}
\end{array}\right]_{n^{2} \times k}
$$

step 2. Whiten the data using Eqs.(3) and (4), where the size of $D$ and $E$ are $n^{2} \times n^{2}$. In our research, we used PCA whitening matrix $V$ to reduce the dimensionality of the image data, which lowers the training time. Assume the dimension of $m$ can be expressed $90 \%$ of the whole data, so the dimensions of $D$ and $E$ become $m \times m$ and $n^{2} \times m$ respectively.

$$
y=W z=W V x=W D^{-1 / 2} E^{T} x
$$

Where $W_{m \times m}, D_{m \times m}$ and $\left(E^{T}\right)_{m \times n^{2}}$

step 3. Give an initial random vector $w_{p} \cdot w_{p}{ }^{\mathrm{T}}$ is the row vector of $W \cdot p=1,2, \ldots, m$

step 4. Compute $w_{p}$ until converged.

$$
w_{p}(t+1)=E\left\{z g\left(w_{p}(t)^{T} z\right)-g^{\prime}\left(w_{p}(t)^{T} z\right) w_{p}(t)\right\}
$$


Where $g=\tanh \left(a_{1} y\right), a_{1}=1$ and $t$ is an iteration number.

step 5. If not converged, go back step 4.

step 6. Do the following orthogonalization to prevent the different vector converged to the same direction.

$$
w_{p}=w_{p}-\sum_{j=1}^{m-1}\left(w_{p}^{T} w_{j}\right) w_{j}
$$

step 7. Normalize $w_{p}$ by dividing it by its norm.

$$
w_{p}=\frac{w_{p}}{\left\|w_{p}\right\|}
$$

step 8. $p=p+1$, go back to step 3, until $p>m$.

step 9. ICs, de-mixing matrix $W$ and Average IC can be obtained.

$$
\begin{gathered}
W=\left[\begin{array}{cccc}
w_{1} & w_{2} & \ldots & w_{m}
\end{array}\right]^{T}, \quad y=W z \\
y_{\text {mean }}=\sum_{p=1}^{m} y_{p}
\end{gathered}
$$

\subsection{ICA detection}

step 1. Test image is also partitioned into non-overlapping sub-images of size $n \times n$ and amount $l$. Each sub-image is converted into a column vector of size $\mathrm{n}^{2} \times 1$ described as below:

$$
x=\left[\begin{array}{llll}
x_{1} & x_{2} & \ldots & x_{l}
\end{array}\right]_{n^{2} \times l}
$$

step 2. ICs of test image can be compute using Equation (5).

$$
y_{i}=W D^{-1 / 2} E^{T} x_{i}, \quad i=1,2, \ldots, l
$$

step 3. Compute each spatial distances $d_{i}\left(d_{i}\right.$ arranged in ascending order) between $y_{\text {mean }}$ and $y_{i}$.

$$
\begin{aligned}
& \text { ascending order }\left[d_{i}\right] \\
& \text { where } d_{i}=\sqrt{\left(y_{\text {mean }}-y_{i}\right)^{T}\left(y_{\text {mean }}-y_{i}\right)}, i=1,2, \ldots, l
\end{aligned}
$$

step 4. Classify each test sub-image as defective or non-defective if $d_{i}$ exceeds a threshold value $T_{d}$.

$$
\left\{\begin{array}{cc}
\text { defetive region } & \text { if } d_{i}>T_{d}, \mathrm{i}=1,2, \ldots, l \\
\text { non-defective region } & \text { otherwise }
\end{array}\right.
$$

The threshold value is determined by the formula $T_{d}=D_{\text {median }}+\eta Q . D$., where $D_{\text {median }}$ and $Q . D$. are the mean of distance and the quartile deviation respectively. 


\subsection{Mura evalution}

Mura is a non-uniform brightness region which merely differs form the background and can be identified by human eyes. During detection process, we have to add mura evaluation which have been proposed as Equation (17) according to the SEMU index defined by SEMI (2002)[1]. From Fig. 2, the luminance brightness of each region can be simplified to the mean of the gray intensity of the corresponding region and will be denoted by $I_{o}$ and $I_{b}$ for the object and the object-background. The flow chart of mura inspection is shown in Fig. 3.

$$
\mathrm{SEMU}=\frac{\left|C_{x}\right|}{C_{\text {jnd }}}=\frac{\left|I_{o}-I_{b}\right|}{\frac{1.97}{S^{0.33}}+0.72}
$$

$I_{o}$ : Average brightness of mura object

$I_{b}$ : Average brightness of object-background

$S$ : Area of mura

$C_{x}$ : Average contrast of mura being measured

(Unit: \% relative to background $100 \%$ )

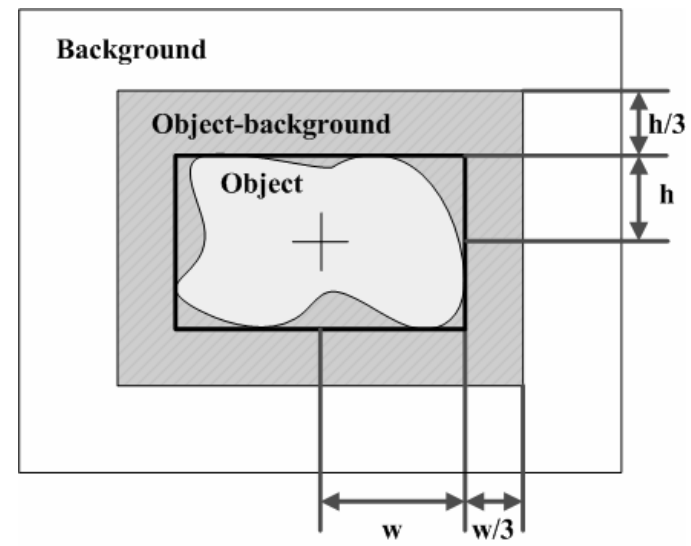

Fig. 2. Mura evaluation regions

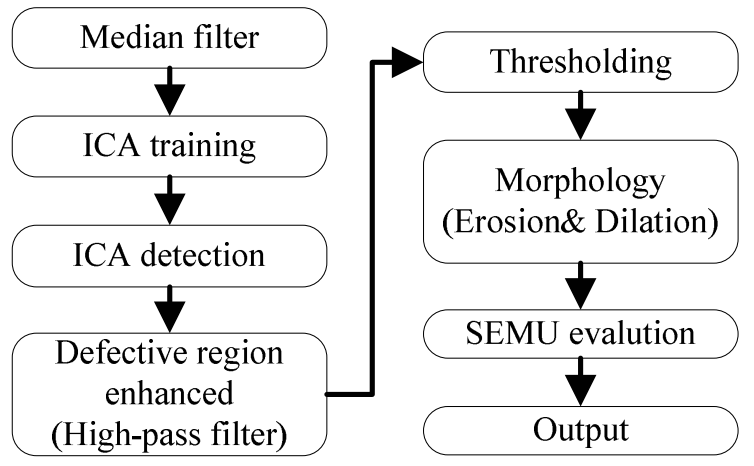

Fig.3 Flow chart of Mura inspection

\section{Experimental Results and Discussions}

Mura defect which differs from background brightness is taken as the experimental image shown in Fig. 1. The pattern generator that generates the image for inspection is supported by TFT-LCD maker. The camera specifications used in this research are shown as below: Industrial camera: Color Area Scan 1/1.8" CCD, 2M pixels; Lens: 18-108 mm; Video capture card: Camera link PCI frame grabber, 64-bit $66 \mathrm{MHz}$ bus, 24-bit $66 \mathrm{MHz}$ Max-pixel-clock frequency; Computer: Pentium 2.8 G, 512MB. 


\subsection{Practical Mura inspection application}

Since background of mura image only has gray level intensity data, it may cause impulse noise of the white or the black pixels because of surroundings light or CCD temperature. In order to make ICA obtain better training ability, the noise must be filtered out through a median filter first. Then there are 100 non-overlapping sub-images $(40 \times 40)$ selected from the non-defective image to be our training samples, which are converted the column vector row by row respectively. Hence $x$ can be expressed as $x=\left[x_{1} x_{2} \ldots x_{100}\right]_{1600 \times 100}$. the training steps are described as the section 3.1. Two practical mura images which are expressed \#1 and \#2 are taken as experiment images shown in Fig. 1. Since mura area could varied in size, thus we chose the sub-window of size $40 \times 40$ with 20 pixels overlapped to detect. The bar graphs of IC vectors of non-defective and defective are shown in Fig. 4. From Fig. 4, we know the defective region has the higher coefficient values than non-defective regions. The defective regions detected by ICA are shown in Fig. 5.

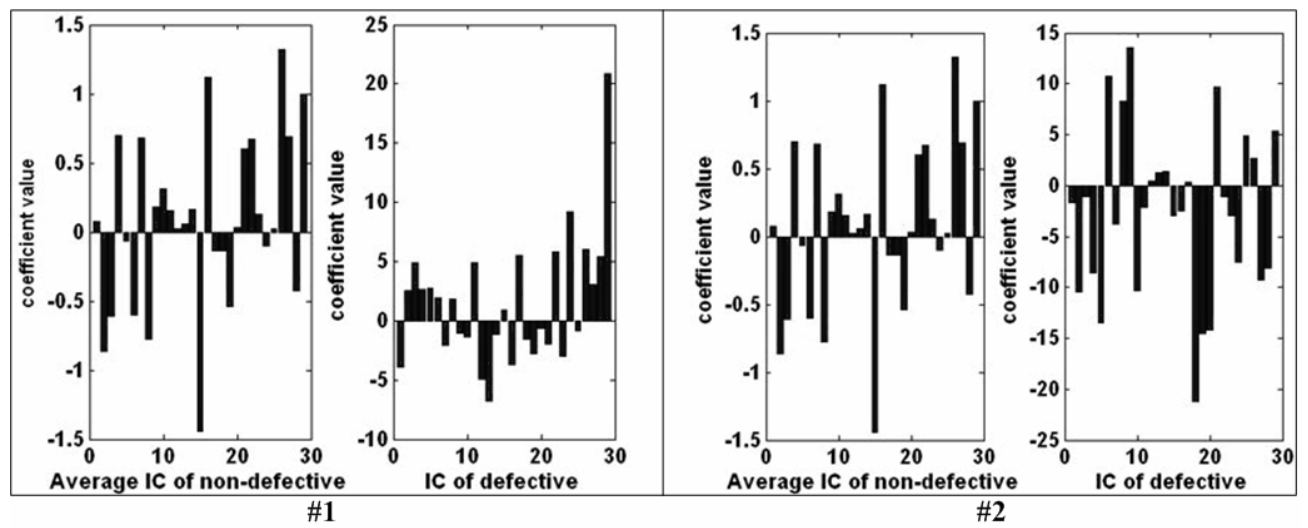

Fig. 4. Bar graphs of ICs
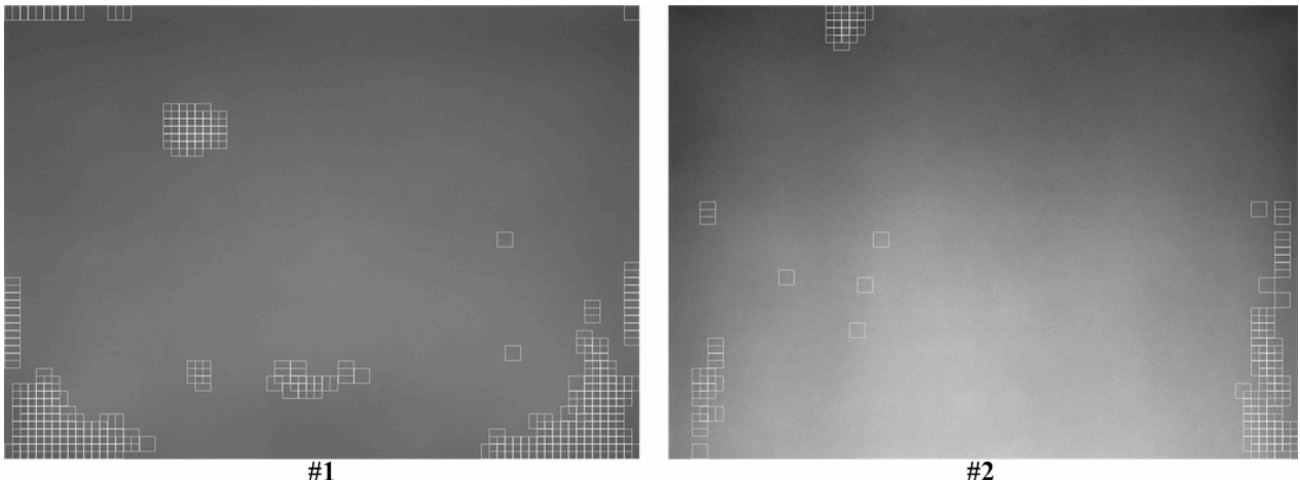

Fig. 5. White rectangles(size: $40 \times 40)$ are the locations of defects. $(\eta: 2$, image size: $1200 \times$ 1600)

After detecting the defective regions via the differences of Euclidean distance between ICs of non-defective and defective, we used high-pass filter to enhance mura gray intensity such that easily segmented the defective regions into binary images individually. Discriminant criteria method proposed by Otsu (1979)[9] is used to be our threshold method. In order to remove excess noise and fill in the gaps of the defects segmented by threshold, we used a rectangle structure element with a radius of 2 pixels to do erosion and dilation. We named the defects within binary image after thresholding as the candidate mura defects, and the next step is to add SEMU index to identify the real mura defects form the candidate mura defects. The SEMU threshold can be adjusted according to the quality demand criteria or the desired recognition threshold of mura contrast. The SEMU value distribution of the candidate mura defects is shown in Fig. 6. In our research, when the SEMU value is larger than 5, it is considered a mura defect which is circled with a rectangle region shown in Figs. 7, 8 the red rectangle regions show the location of original image corresponding to mura defects. Table 1 shows ICA inspection accuracy relative to human vision. From above mentioned, we prove ICA is a effective method similar with human vision for mura inspection. 


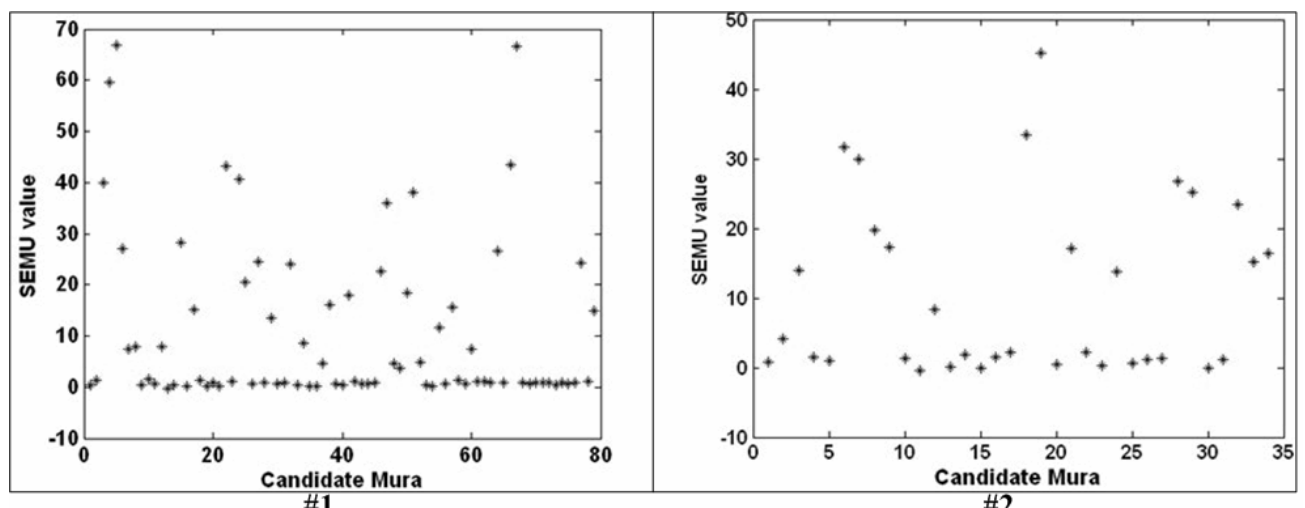

Fig. 6. Distribution of SEMU value of candidate mura

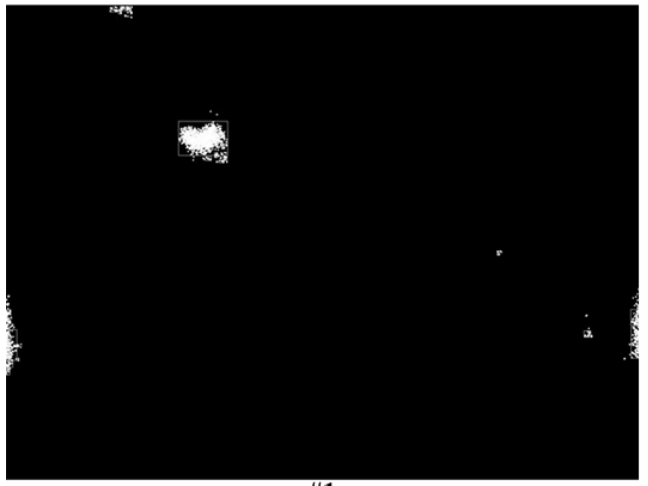

\#1

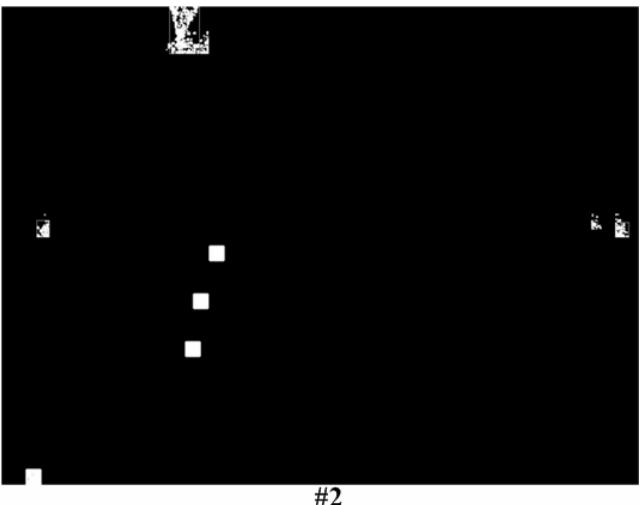

\#2

Fig. 7. Binary images of Figure 5. (image size: $1200 \times 1600$ )

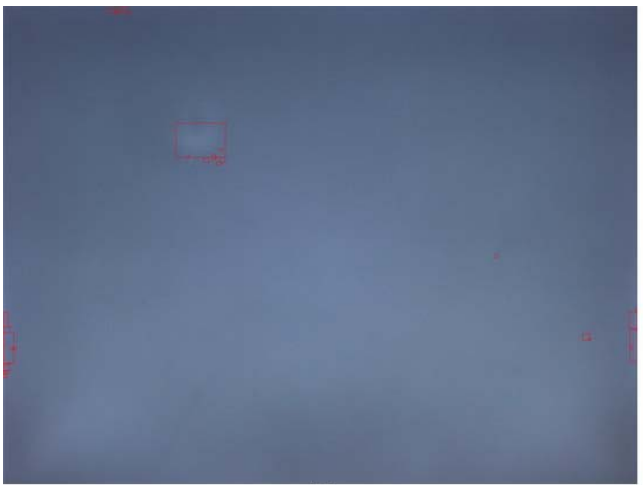

\#1

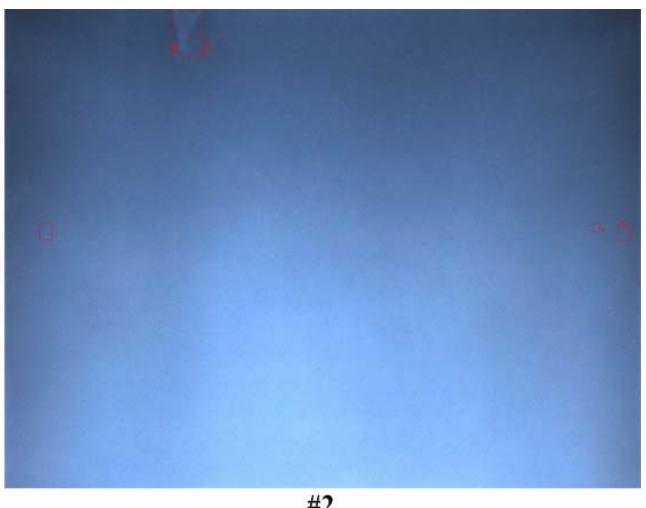

$\# 2$

Fig. 8. Mura defects corresponding to the locations of Figure 3. (image size: $1200 \times 1600$ ) 
Table 1. Experiment Comparsion of ICA and human vision

\begin{tabular}{|c|c|c|c|c|c|}
\hline \multirow{2}{*}{$\begin{array}{c}\# \text { of } \\
\text { experimental } \\
\text { images }\end{array}$} & \multicolumn{2}{|c|}{ Human vision } & \multicolumn{2}{|c|}{ ICA } & \multirow{2}{*}{$\begin{array}{l}\text { ICAinspection } \\
\text { accuracy (relative } \\
\text { to human } \\
\text { vision, } \% \text { ) }\end{array}$} \\
\hline & $\begin{array}{c}\text { Mura } \\
\text { amount }\end{array}$ & $\begin{array}{l}\text { Mura } \\
\text { location }\end{array}$ & $\begin{array}{c}\text { Candidate } \\
\text { Mura amount }\end{array}$ & $\begin{array}{c}\text { Mura } \\
\text { amount }\end{array}$ & \\
\hline$\# 1$ & 1 & Upper left & 79 & 30 & $100 \%$ \\
\hline$\# 2$ & 1 & Upper left & 34 & 15 & $100 \%$ \\
\hline
\end{tabular}

\subsection{Correctness Assessment}

To verify the practicality of this method, we also used ICA to detect artificial blob mura which was simulated using Gaussian function as Equation (18). Since mura defect is a non-uniform brightness region which merely differs form the background, thus we simulated 24 bright blob mura defects with the different size and brightness. The gray peak values of 24 blob mura defects are sorted in ascending order from 1 to 24 pixels. The simulated mura image is shown in Fig. 9(a) which is acquired practically by an area camera with 24-bit brightness resolutions. Fig. 9(b) the white rectangle regions with the size of 40 $\times 40$ are the defects detected by ICA. A total of 18 blob mura defects were found and a total of 6 undetected blob mura with small size and the gray level differences of 1-6 pixels were not found. Fig. 9(c) is a binary image. Fig. 9(d) is a comparison of the location between detected blob mura and the original image. Fig. 10 is the distribution of SEMU value. The locations of 18 detected blob mura are inside the red rectangle region.

$$
g(x, y)=a e^{-\gamma^{2} / 2 \sigma^{2}}
$$

$a:$ Constant

$\gamma:$ Radius of mura

$\sigma:$ Adjust the gray intensity with the radius decreased

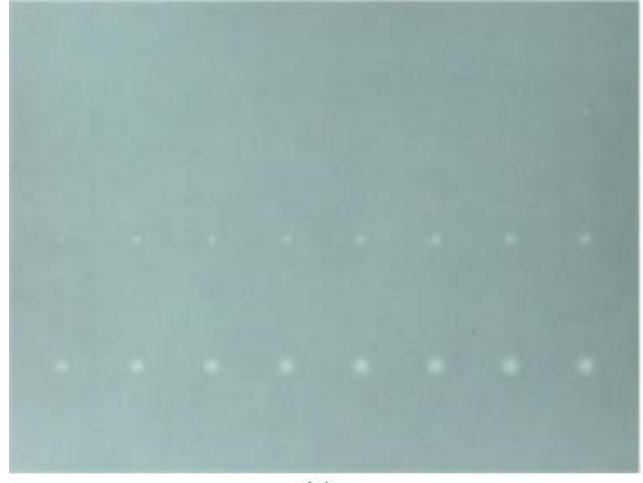

(a)

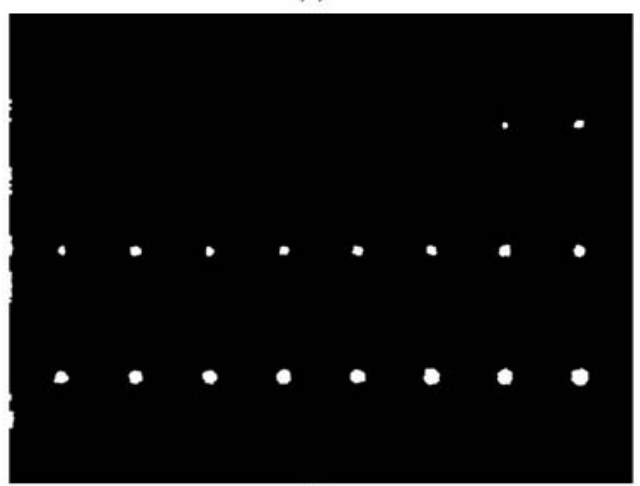

(c)

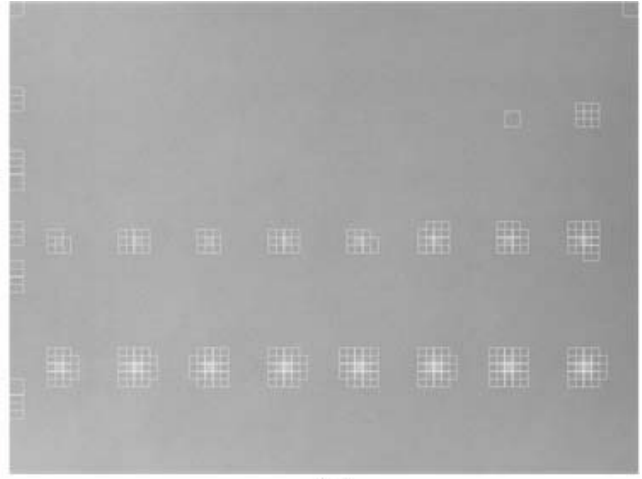

(b)

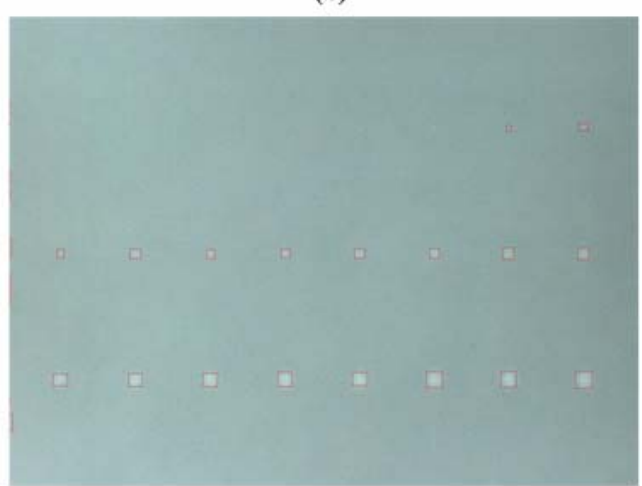

(d)

Fig. 9. Output images during the different of inspection processes. (image size: $1200 \times 1600$ ) 


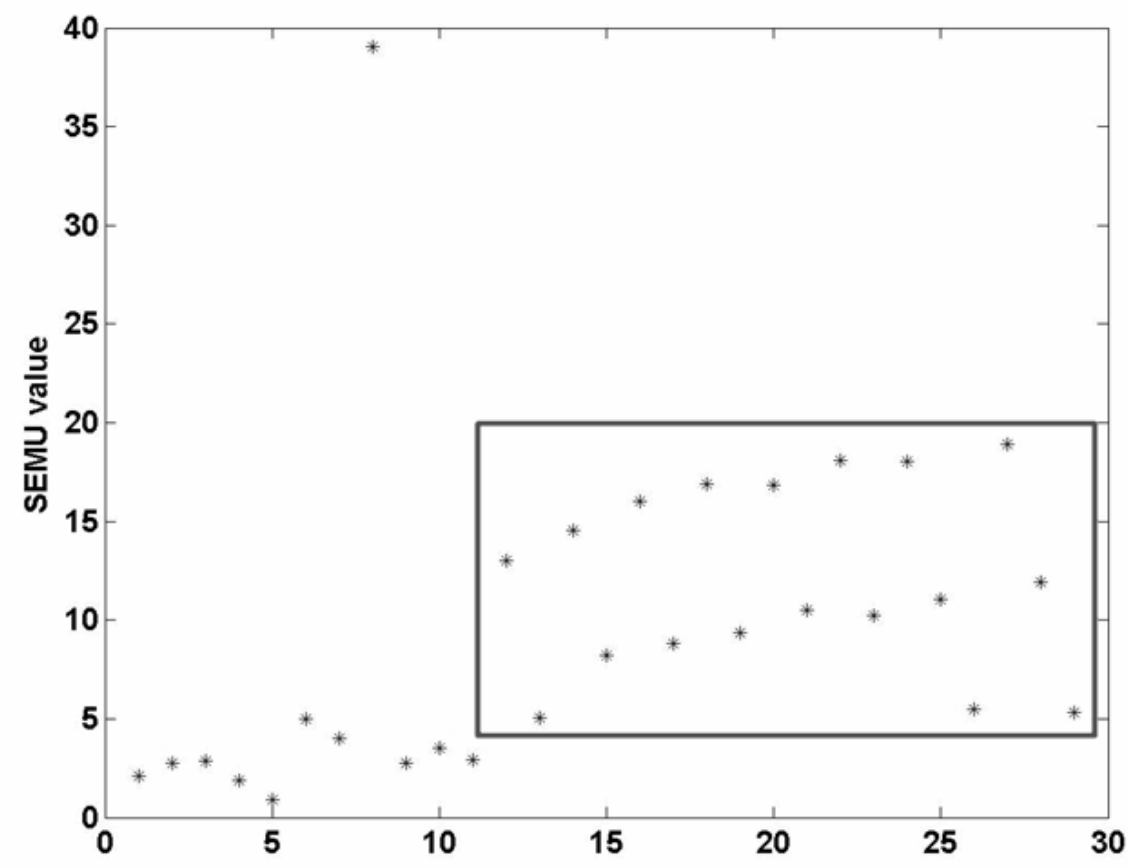

Fig. 10. Distribution of SEMU value. Each SEMU value of 18 detected artificial Mura defects are inside the red rectangle region.

\section{Conclusions}

This research applied independent component analysis in mura defects inspection of TFT-LCD, and the conclusions were as following:

A. If the image histogram is close to unimodal, this makes image segmentation difficult. In this research, we used ICA to detect defective regions before thresholding in order to avoid excess influence from background and noise.

B. The experimental parameter settings as sample size, sample amount and $\eta$ value influenced the inspection performance during training or detection process. The smaller the size, the more ineffectiveness in describing the background features; the larger the size, the lower the effect of defects inspection. The larger the amount of samples, the more training time it takes; the smaller the amount of samples, the poorer the ICA training results. The selection of $\eta$ could be used to reduce the inspection false-positive ratio. From above mentioned, the parameters which are the sample size of $40 \times 40$, the total sample number of 100 and $\eta$ of 2.2 have better performance for mura inspection.

C. In the experiment of non-uniform background image inspection, this research not only detected mura defects but also artificial mura defects to verify the practicality of this method. We prove ICA is effective in detection of large mura defects.

D. besides texture segmentation or texture defects inspection in general, ICA which applied in non-uniform background inspection also have good effect, and mura evaluation depends on the desired recognition threshold of mura contrast.

\section{Acknowledgements}

Part of the research results are supported by NSC (NSC 95-2221-E-006-400). The financial supports are gratefully acknowledged.

\section{References}

[1] SEMI, 2002. New standard: Definition of measurement index (SEMU) for luminance Mura in FPD image quality inspection. Semiconductor Equipment and Materials 
International (SEMI) standard, 4113 242-249.

[2] Lee J. Y. and Yoo S. I., 2004. Automatic Detection of Region-Mura Defect in TFT-LCD. IEICE TRANSACTIONS on Information and Systems, 87 (10), 2371-2378.

[3] Nakano H. and Mori Y., 2005. Measurement method for low-contrast nonuniformity in liquid crystal displays by using multi-wavelet analysis. Proceedings of SPIE, 5880 588013.

[4] Lu C. J., 2004. Automatic defect inspection for patterned TFT-LCD panel surfaces using singular values decomposition and independent component analysis. Yuan Ze University.

[5] Jenssen R. and Eltoft T., 2003. Independent component analysis for texture segmentation. Pattern Recognition, 10 2301-2315.

[6] Amet A. L., Ertuzun A., and Ercil A., 1998. Texture defect detection using subband domain co-occurrencematrices. IEEE Southwest Symposium on Image Analysis and Interpretation, 205-210.

[7] Sezer O. G., Ertuzun A., and Ercil A., 2004. Independent Component Analysis for Texture Defect Detection. Pattern Recognition and Image Analysis, 14 (2), 303-307.

[8] Hyvarinen A., 1999. Fast and robust fixed-point algorithms for independent component analysis. Neural Networks, IEEE Transactions on, 10 (3), 626-634.

[9] Otsu N., 1979. A threshold selection method from gray level. IEEE Transactions on Systems, Man, and Cybernetics, 9 (1), 62-66. 